\title{
Use of the Multi-Agent Paradigm in Sustainable Tourism
}

\author{
Martina Husáková \\ e-mail: martina.husakova.2@uhk.cz
}

Department of Information Technologies, Faculty of Informatics and Management, University of Hradec Králové, Hradec Králové, Czech Republic

Husáková, M. (2018). Use of the Multi-Agent Paradigm in Sustainable Tourism. Czech Journal of Tourism, 7(1), 5-24.

DOI: 10.1515/cjot-2018-0001.

\begin{abstract}
Complex systems are characterised by a huge amount of components, which are highly linked with each other. Tourism is one of the examples of complex systems collecting various activities leading to the enrichment of travellers in the view of receiving new experiences and increasing economic prosperity of specific destinations. The complex systems can be investigated with various bottom-up and top-down approaches. The multi-agent-based modelling is the bottom-up approach that is focused on the representation of individual entities for the exploration of possible interactions among them and their effects on surrounding environments. These systems are able to integrate knowledge of socio-cultural, economic, physical, biological or environmental systems for in-silico models development, which can be used for experimentation with a system. The main aim of the presented text is to introduce links between tourism, complexity and to advocate usefulness of the multi-agent-based systems for the exploration of tourism and its sustainability. The evaluation of suitability of the multi-agent systems in tourism is based on the investigation of fundamental characteristics of these two systems and on the review of specific applications of the multi-agent systems in sustainable tourism.
\end{abstract}

\section{Keywords}

tourism, sustainability, modelling, simulation, complexity, multi-agent system

JEL classification: C53, C63, L83 


\section{Introduction}

Tourism is one of the forms of leisure time activity, which is mainly related to free time, travelling, relaxation, discovery of new places, cultures and habits, spending money or doing sports. Everyday activities are often fulfilled by work. Therefore, it should be natural to receive new energy and enrich life with new experiences. Travelling for fun can ensure psychological and physical homeostasis. Tourism is not limited only to leisure, but it can include work-related activities. It depends how one defines tourism. It is difficult to define tourism precisely and completely. Historically, definitions of tourism were different from each other in the view of the amount of hours of travelling and staying outside of one's own residence and reasons of travelling (business, meetings, or visits of family members or relatives). One of the most cited definitions of tourism comes from the UNEP and UNWTO (the United Nations Environment Programme and the United Nations World Tourism Organisation): “Tourism comprises the activities of persons travelling to and staying in places outside their usual environment for not more than one consecutive year for leisure, business and other purposes" (UNEP \& UNWTO, 2005; Holloway, Humphreys, \& Davidson, 2009). One of the most cited issue, regarding tourism, is its sustainability. Sustainability of tourism is a term appearing today mainly due to its possible negative effects, especially on natural environment and resources, cultural heritage, immigration or economic prosperity. Sustainable tourism is defined by The United Nations World Tourism Organisation UNWTO (UNEP \& UNWTO 2005) as follows: "Tourism that takes full account of its current and future economic, social and environmental impacts, addressing the needs of visitors, the industry, the environmental and host communities." The main aim of sustainable tourism is to minimise its negative effects and to establish a balance among various systems on which tourism has the impact. The development of sustainable tourism is a continuous and complex long-term activity requiring training and education, monitoring of impacts of tourism on societies and provision of effective strategies for management of sustainable tourism. Testing of various scenarios for sustaining tourism with minimum negative impacts on society in real environment is a time-consuming activity, often burdened with high costs and negative impacts on the environment or natural sources. Modelling and simulations are alternatives for the investigation of less or more complex systems with minimum spending time and costs. Simulation models explore a system under different conditions and test various hypotheses about its possible behaviour. The multi-agent system is one of the bottom-up modelling approaches able to model dynamic complex processes. The main aim of the following text is to advocate the usability of the multi-agent systems in sustainable tourism and to present interesting and useful applications of the multi-agent systems in tourism with respect to the use of these solutions in different application areas, and the periods when the research studies were published. Before the exploration of possible relations between the multi-agent systems and sustainable tourism, the theoretical background has to be clarified. 


\section{Tourism as a complex system}

The Complex Systems are focused on the investigation of relations among various parts of a system and the explanation of how these relations give rise to collective behaviour of the system and how the system interacts and forms relationships with its environment. The research of the complex systems is highly multi-disciplinary. It interconnects mathematics, statistics, systems theory, computer science (artificial or computational intelligence), natural or social science. Tourism is an open system consisting of two general components - demand (tourist) and supply (tourism agency). These components exist in a specific environment enabling to make contracts among the interested parties, see Fig. 1.

Figure 1 System view on tourism

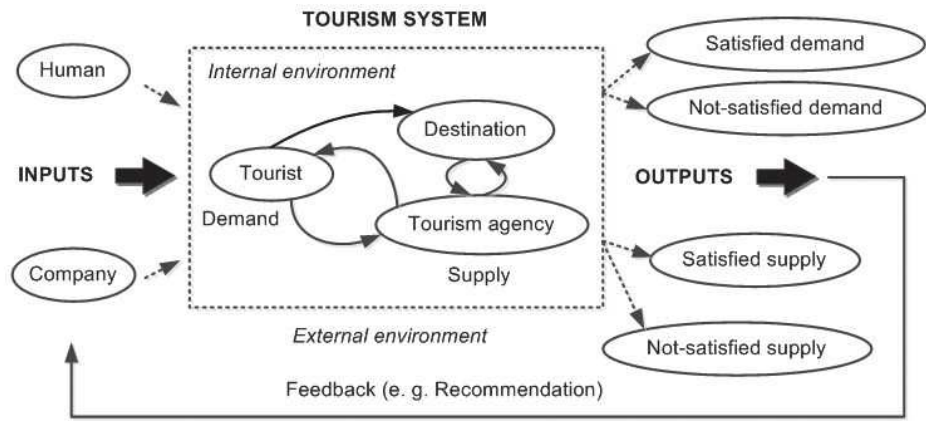

Source: own processing

The environment specifies the rules (restrictions) and conditions which affect demand and supply. Tourism can be perceived as a complex system because of the amount of the interested 'players' and interactions existing among them. There is not any generally accepted definition of the complex system, but some share similar characteristics. Three definitions, including various facts, are selected from various periods:

- “... a system is said to be complex when it exhibits some type of order as a result of the interactions of many heterogeneous objects" (Durlauf, 1998),

- '... the scientific community has coined the rubric 'complex system' to describe phenomena, structure, aggregates, organisms, or problems that share some common theme: (i) They are inherently complicated or intricate [...]; (ii) they are rarely completely deterministic; (iii) mathematical models of the system are usually complex and involve non-linear, ill-posed, or chaotic behaviour; $(i v)$ the systems are predisposed to unexpected outcomes (the so-called emergent behaviour)." (Foote, 2007),

- "When systems are complex, their structures cannot be described at a single level or with a single view; multi-scale descriptions are needed to understand complex systems. Their emergent 
behaviour, derived from the relationships among their elements and with the environment, via internal and external feedback loops, gives rise to observed patterns that may not be understood or predicted." (Sheard et. al, 2015).

The complex systems share some common attributes. The following attributes are distinguished for the complex systems according to Baggio (2005) and Miguel et al. (2012) and corresponding examples related to tourism are mentioned in Tab. 1 below.

Table 1 Complexity and tourism

\begin{tabular}{|c|c|c|}
\hline & $\begin{array}{l}\text { Characteristics of the } \\
\text { complex systems }\end{array}$ & Tourism-based examples \\
\hline \multirow{3}{*}{ 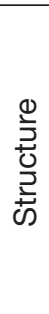 } & $\begin{array}{l}\text { Many heterogeneous } \\
\text { interacting parts }\end{array}$ & $\begin{array}{l}\text { tourists, companies (travelling agencies, kinds of } \\
\text { accommodation, financial institutions, carriers), destinations, } \\
\text { environments }\end{array}$ \\
\hline & Il-defined boundaries & pollution, terrorism, markets \\
\hline & $\begin{array}{l}\text { Distributed nature of the } \\
\text { system }\end{array}$ & $\begin{array}{l}\text { no global control in tourism, network-based architecture of } \\
\text { tourism }\end{array}$ \\
\hline \multirow{6}{*}{ 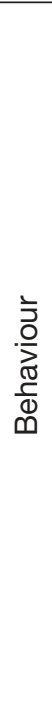 } & $\begin{array}{l}\text { Emergence } \\
\text { (unpredictable) }\end{array}$ & $\begin{array}{l}\text { weather, catastrophes, terrorism, fluctuations in markets or } \\
\text { political scene, changes in prices, mobility of immigrants }\end{array}$ \\
\hline & Self-organisation & strikes, demonstrations, immigration \\
\hline & Non-determinism & $\begin{array}{l}\text { weather in general, environmental events (earthquakes, floods), } \\
\text { viruses or spread of illnesses }\end{array}$ \\
\hline & $\begin{array}{l}\text { Presence of feedback } \\
\text { loops }\end{array}$ & $\begin{array}{l}\text { interactions between supply and demand in general can } \\
\text { influence the future trading (recommendations about } \\
\text { experience with specific travel agency can be shared and } \\
\text { distributed to the new potential tourists) }\end{array}$ \\
\hline & $\begin{array}{l}\text { Adaptability to changing } \\
\text { environment }\end{array}$ & $\begin{array}{l}\text { presence of serious environmental events should influence } \\
\text { specification of counter measures }\end{array}$ \\
\hline & $\begin{array}{l}\text { Sensitive dependence } \\
\text { on initial conditions }\end{array}$ & weather, traffic (jams), changes in prices in stock market \\
\hline
\end{tabular}

Source: own processing

According to the facts mentioned above, Fig. 1 is slightly modified, see Fig. 2. Complexity of tourism system is determined by the network-based and distributed architecture consisting of many interacting entities (travellers, companies, travel agencies, companies providing various types of accommodation, financial institutions, or transport com- 
panies), possible feedback loops and communication channels existing between them. Tourism significantly influences and is influenced by other highly complex systems, i.e., an economic system, a socio-cultural system and an environmental system of particular country, which are interconnected with each other and influence each other. It is obvious that tourism cannot be perceived as an isolated system.

Figure 2 Complexity and tourism

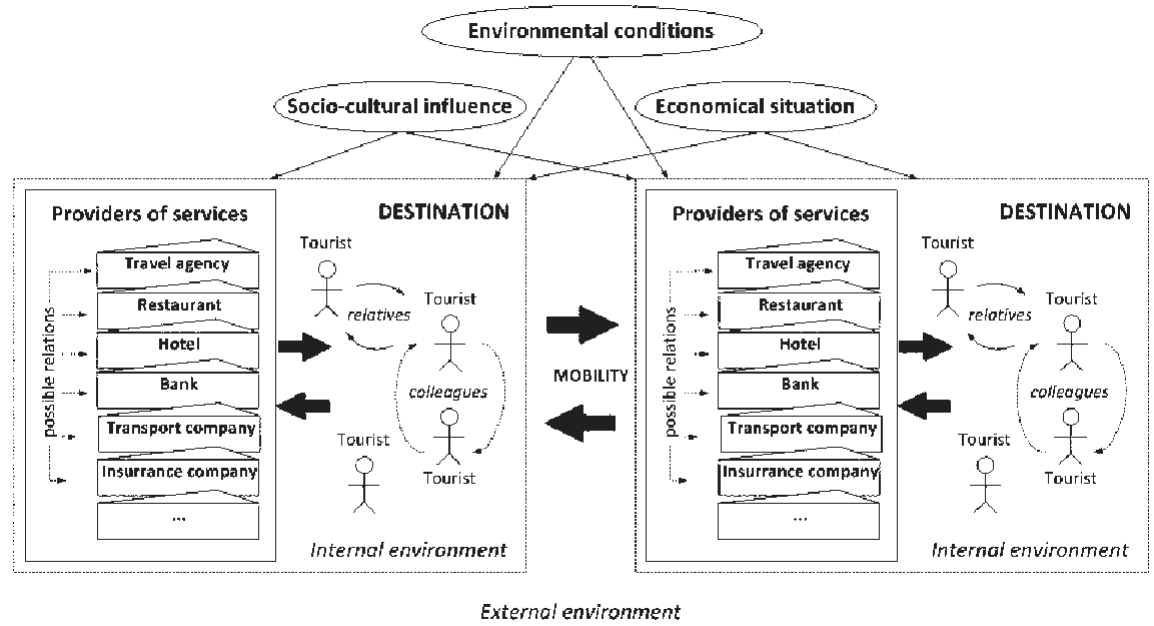

Source: own processing

The complex systems modelling and simulation are the approaches to better understanding, explanation and prediction of behaviour of more or less complex systems. They are the methods using a system approach to and thinking about building of models which are the outputs of the modelling process. The multi-agent models and simulations play an important role in the methods for doing research (Axelrod, 2003). This kind of the model is perceived as a computational model representing individual elements (agents) of a real system and relations among them, especially for explanation, understanding and prediction of behaviour of a real system. The multi-agent systems (MAS) bring a promising approach for the exploration of a tourism complex system, e.g., because of a close parallel between the interacting entities in tourism and the notion of the artificial agents/agencies used in MAS. The following parts of the text introduce the multi-agent systems; firstly, in the context of the artificial intelligence, and secondly, in the context of tourism. 


\section{Complex systems modelling with the multi-agent systems}

History of the multi-agent systems begins with distributed artificial intelligence (DAI). The distributed artificial intelligence is a sub-area of artificial intelligence which started to develop in seventies. The main aim of DAI is to investigate principles and mechanisms which should ensure the solution to problems similar to human. One of the most important questions that was raised by Weiss (2000) is: "When and how should agents interact - cooperate and compete - to successfully meet their design objectives?" This question is important also at present. Researchers deal with this question in two main branches of DAI whose frontiers are not strictly distinguished - distributed problem solving and multi-agent systems. The distributed problem solving is focused on efficient and intelligent allocation of individual tasks among more autonomous intelligent entities (modules, nodes, agents). These entities should be able to cooperate with each other and use their knowledge for goal fulfilment. The multi-agent systems investigate various ways of cooperation, coordination and communication of autonomous intelligent agents, i.e., how the agents should use them with their abilities, knowledge, believes, plans, intentions for decision making, and problem solving.

\section{Agent}

A generally accepted definition of an agent does not exist because of different views of their authors and various applications where the agents can be applied. One of the most cited definitions is mentioned by Wooldridge and Jennings (1995) - initiators of the intelligent agent paradigm (in Wooldridge, 2013): "An agent is a computer system that is situated in some environment, and that is capable of autonomous action in this environment in order to achieve its delegated objectives." This definition takes into account only artificial systems as agents. This is too restricted view and can be extended. According to Mills (2010): “An 'agent' is the part of a model that represents an actor within a system. It might represent a person, an organization, an economic sector such as health care, a governmental entity, or a country." Generally speaking, an agent can be artificial or biological system which is able to autonomously (without human intervention) solve specific problems using its sensors (perceptors), internal architecture and effectors (actuators). The sensors are used for sensing the environment (virtual or real) and collecting data which are then processed by the algorithms contained in the internal architecture of the agent. The effectors provide reactions and feedback, which can change the state or behaviour of other agents, and the environment as a whole, see Fig. 3. The sensors and effectors enable to interact with the other agents, i.e., to coordinate, cooperate and communicate in order to achieve the specific objectives delegated to them.

The ability to interact and communicate with the environment is a specific sign of the sociability of the agent. The sociability of the agents is not manifested only in the cooperation, but also in the competitive tendencies. From this point of view, one can distinguish two groups of agents: pro-active and self-interested (Vig \& Adams, 2006). The pro-active agents preserve the interests of the group to which the agent belongs. 
Figure 3 Complexity and agent

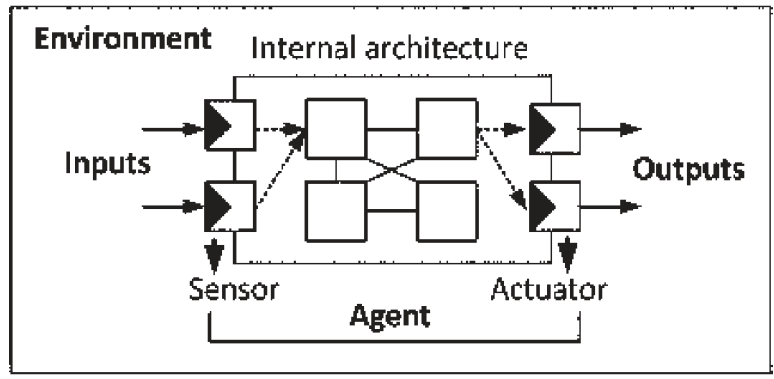

\section{Source: own processing}

The self-interested agents preserve only their own interests which can be in contradiction with other agents in the society. The environment plays the crucial role in 'lives of agents'. The agents are situated in a specific environment providing infrastructure and conditions for interactions and communication. It can play a role of a 'shared memory', where the messages can be stored and received by other agents in the future.

The sophistication of agent problem solving depends on the architecture of the agent, which determines its type. There are various classifications of the intelligent agents. One of them has been already presented in the previous paragraph (pro-social/self-interested agents). Wooldridge (2013) distinguishes the following categories of agents: reactive agents, deliberative agents, social agents and hybrid agents. The reactive agents are based on the stimulus - reaction model. These agents use sensors (perceptors) for sensing the environment and receiving stimuli. The effectors (actuators) are used for responding to stimuli. These agents can use an internal memory for storing their internal states. The reactive agents are not able to fulfil goals according to a specific plan. The architecture of the reactive agents is very simple, but complex and often unexpected behaviour can occur with an inclusion of more reactive agents with these simple architectures. The social insects are typical examples of agents using simple rules for decision making. These simple rules can lead to finding the shortest path towards a food source. The deliberative agents are based on the stimulus - plan - reaction model. This type of agents is able to use a memory for explicit representation of the environment and planning of their activities for problem solving and decision making. BDI (Belief-Desire-Intention) theory is mainly used for modelling of their mental processes. The social agents have sophisticated mechanisms of communication. These agents are often grouped into social groups, similarly to humans in interest groups. They are able to cooperate with each other and to receive benefits from cooperation. The hybrid version of an agent is a mixture of the above mentioned characteristics of agents. 


\section{Multi-agent system}

The agent system can be defined differently by different authors. This is also true for a multi-agent system where its explanation shares similar attributes in various literature sources. Wooldridge (2013) explains that in the following way "a multi-agent system is a system composed of multiple interacting intelligent agents that interact to solve problems that are beyond the individual capabilities of knowledge of each individual." From this point of view, agents of a multi-agent system can be homogeneous or heterogeneous. The homogeneous agents share the same characteristics which can be used for decision making. The homogeneity can lead to robustness of the system in which one part of the system can be substituted by the second part of the system. The heterogeneous agents dispose of a wider variety of abilities, i.e., if one agent is not able to complete a task, another agent can do that. The organisational paradigms of MAS prescribe rules for behaviour of agents or groups of agents, i.e., an intensity of communication (less or more intensive communication), overlapping of groups of agents in the view of abilities (roles), openness of a group of agents, a level of freedom for entering or leaving of agents into/from MAS, duration of an organisation structure or types of agents (according to the homogeneity and heterogeneity). According to these attributes, several organisational paradigms exist. Horling and Lesser (2004) distinguish the following organisational paradigms: hierarchy, holarchy, coalition, team, congregation, team, society, federation, market, matrix organisation and compound organisation, for more details, see Horling and Lesser (2004).

The multi-agent systems can be built with various development environments, which can be classified according to different criteria. Basically, the multi-agent systems can be programmed with 'complete' development platforms providing options for the management of projects, creation and editing of source files, testing, building and running a model, often including tools for a fundamental data analysis; e.g., NetLogo (Wilensky, 1999), Repast Simphony (Collier et al., 2003), or AnyLogic (Borshchev, 2013). Libraries are the second possibility. They contain packages for MAS development; e.g., MASON (Luke et al., 2005). An overview of various MAS-based environments can be found, e.g., in Kravari and Bassiliades (2015) where a detailed comparison of tools is mentioned.

\section{Applications of the multi-agent systems in tourism}

The following section of the text introduces interesting and useful applications of the multi-agent systems in tourism. These applications are selected according to the various areas of their usage and various periods, i.e., from 2006 to 2016. These applications are ordered according to their purpose in the text below, but individual research studies can be classified into more sections. Tab. 2 below summarises the selected studies discussing how MAS can be used in tourism. The studies are ordered chronologically. 


\section{Frameworks and methodologies}

The frameworks and methodologies propose guidelines for the system development and they often facilitate the development process through the whole system life cycle. The agent-based model of movement of tourists in New Zealand is proposed in (Doscher et al., 2011). The methodology for transferring the qualitative data, received from interviews with tourists, into decision-making heuristics is proposed. The interviews were collected because of the identification of reasons for the selection of a specific destination and its attributes by tourists. The conclusions from the interviews were used for the agent-based model development. Various conditions (weather, earthquakes, volcanic eruptions) influencing the movements of tourists were simulated in Repast Simphony in the case study (Colier et al., 2003).

Marguez, Espinoza-Hernandez, and Magdaleno-Palencia (2012) propose a different methodology using various techniques for modelling the complex systems, which are not normally used for the investigation of societies. The methodology is applied for a study of urban growth of the Mexican city Ciudad Juarez. The main aim of the simulation is to investigate sustainability of urban growth in the city. Sustainability is analysed with the top-down (system dynamics) and bottom-up (agent-based) approach with NetLogo tool (Wilensky, 1999).

The agent-based migration framework is proposed by Kaur, Kahlon, and Virk (2014). The framework is applied for modelling migration of artificial agents in and among societies (the internal migration and the external migration). The main aim is to build the agent-based models for the explaination of consequences and reasons of migration. The agent-based migration framework is based on the formal definition of a world of agents, population, roles of agents, individual agents and a migration function. All these components influence the migration process, especially "an agent, the society of origin and the destination society" (Kaur, Kahlon, \& Virk, 2014). The presented migration model is based on W5 (Who?, Why?, Where?, What?, When?) social-gravity migration model that is able to model complex migration behaviour in and among communities of agents. This study presents various types of migrations and differences among the migration of agents, humans and animals together with related studies.

\section{Modelling and simulation for the explanation and prediction}

Modelling and simulation is a scientific method used for better understanding, explanation or behaviour prediction of more or less complex systems. Modelling uses a system approach to and thinking about building of the models which are the outputs of modelling process. Simulation uses computational models of the investigated systems for experimentation. The multi-agent models and simulations play an important role in the methods and approaches for doing research (Axelrod, 2003). This kind of the model is perceived as a computational model representing individual elements (agents) of a real system and relations among them, especially for explanation, understanding, and prediction of behaviour of a real system. 
Balbi, Perez, and Giupponi (2010) present their research study in which various scenarios of tourism development in the weather-sensitive European Alpine region are investigated with the agent systems. This region detects increasing mean temperature up to $+2^{\circ} \mathrm{C}$ and this fact influences tourism in the Alpine region. The agent-based model called AuronzoWinSim is a prototype and is aimed at the simulation of responses of tourists under various climate scenarios and at the analysis of various winter development strategies. The model is analysed with the ODD protocol that is primarily used for documentation of various types of models representing some complex phenomena. The UML (Unified Modelling Language) language is applied for conceptualisation of the application domain, i.e., changes in climate and their influence on decision-making by tourists in the Alpine region.

The Galapagos Islands are important eco-touristic destinations which are located in the east of the Pacific Ocean. The agent-based approach is applied by Pizzitutti, Carlos, and Walsh (2014) for the investigation of various changes in tourism in this exotic location. The agent-based system is used for testing of the three main scenarios: if the new airport will be opened, how it will influence tourism (1), how an effect of decreasing the amount of tourists will influence tourism (2), how an effect of increasing a price for parking will influence tourism in the location (3). This research study proves that the outputs of the agent-based simulation are consistent with the available empirical data. The agent-based system was developed in MASON environment (Luke et al., 2005).

It is obvious that weather or climate changes can dramatically influence the interest in a particular touristic location. Winter-ski tourism is one of the most important incomes of Andorra - a region between France and Spain. This is the reason why it is important to analyse the impacts of climatic and weather changes on tourism in this location. The agent-based approach is applied for the analysis of climatic and weather changes and their impacts on winter-ski tourism in Andorra (Pons-Pons et al., 2012). The model should help with the decision-making of businessmen how to choose an appropriate strategy or adapt this strategy in case of climate changes. NetLogo tool (Wilensky, 1999) and GIS support is used for the multi-agent-based system development.

At present, migration is a frequently mentioned word in our society. Migration is highly related to tourism, because it can influence the interest in a particular touristic location. Lin, Carley and Cheng (2016) apply the multi-agent systems for the simulation of migration processes. They developed the agent-based model that is able to represent a possible population shift and migration probabilities among countries. NetLogo 3D (ver. 5.2.0) is used for the agent system development. The introduced review proves that the multi-agent paradigm is able to successfully explain the reasons of migrations, such as climate conditions, economic situation or warlike conditions. Another study introducing the use of the multi-agent systems for the investigation of migration behaviour is mentioned in section 4.1 (Kaur, Kahlon, \& Virk, 2014).

The agent-based systems can also be used for the exploration of how asymmetric information can influence the tourism market (Baggio \& Baggio, 2013). The asymmetric information causes a situation when one side of the market has less information then the second side of the market. This typically happens when the buyer has more information 
about selling products than the customer. Today, the situation can be changed due to the massive use of the internet and web technologies when the customers can have more information than the sellers. Smeral (1993) claims that the asymmetric information can occur also in tourism. The agent-based approach is used because of the possibility to explore structural and dynamical aspects of the complex system. Two scenarios are tested in NetLogo (Wilensky, 1999). The first one considers complete information at the side of the seller and the second considers the complete information on the side of the buyer. The developed NetLogo-based model verifies the economic theory: "When the information asymmetry affects travellers, the market shrinks towards low-quality destinations, while the opposite happens when the level of information travellers have on destinations is higher than destinations have on themselves."

\section{Planning}

Activity planning is substantial for tourism development. The agent-based planning support system called the TourSim is developed for the investigation of tourism in a province of Canada Nova Scotia (Johnson \& Sieber, 2009). The main aim of the TourSim agent-based model is to help policy makers with decision making and planning in tourism, i.e., to simulate interactions between tourists and destinations. The agents are heterogeneous entities differentiating in preferences. The attributes of the tourist agents and various destinations are based on real data. The model helps with answering the 'what-if questions' with the aid of experiments, e.g., the influence of changes on the tourist port of entry in the province. All experiments are conducted in AnyLogic (Borshchev, 2013).

The agent-based planning system for leisure activities of tourists is proposed by Sebastia, Giret, and Garcia (2010). The agents of this system are able to specify a user profile for a tourist and recommend a time schedule for travelling. The paper extends the previous e-Tourism application (Sebastia et al., 2009) which recommends possible activities in the city of Valencia (Spain) on the basis of a user profile. The extension is based on the use of a multi-agent system which should provide more flexibility and adaptability for the e-Tourism application. The multi-agent system uses a tourism ontology describing possible attributes of activities of tourist together with commonly known information about locations and their attributes (types of building, architectonic styles, etc.).

The research study presented by Chao, Furuta, and Kanno (2011) proposes a general framework for the sustainable tourism planning process, because the systematic methodology does not exist for tourism planning. The framework is based on the multiagent systems, GIS and Pattern-Oriented Modelling (POM). POM is a strategy for the investigation of the complex systems. It is based on the identification of patterns which occur in the system and which are important for decoding complexity of the system. It is obvious that tourism is a complex system and authors of the paper believe that the effective tourism planning can be done with the use of the POM-based approach. The general framework is also applied for one case study investigating land use change in 
Hakone - a part of the Fuji-Hakone-Izu National Park, less than 100 kilometres far from Tokyo. The NetLogo-based (Wilensky, 1999) simulation is used for the demonstration of the general framework for sustainable tourism planning.

\section{Recommender systems}

If a tourist wants to discover new destinations, he or she decides according to various criteria which can be contradictory if more decision makers (tourists) choose the destination. The recommender systems can bring an added value because of the ability to predict items that may fulfil tourist needs and expectations. The Social-Net Tourism Recommender System (STRS) is proposed in (Bustos et al., 2009). This system is based on the previous Multi-Agent Tourism System (MATS) (Lopez et al., 2007) providing various services for tourists, e.g., finding information about interesting places (restaurants, movie theatres, museums, or churches), making a reservation in a particular place in a given time period or providing a plan for the whole day in a particular location according to the priorities of the tourist. MATS is extended in (Bustos et al., 2009) with a new trust component for increasing the performance of the recommendation system in terms of accuracy, relevance and quality. STRS combines the multi-agent paradigm with a social network analysis that is used for modelling communities of tourists with the aim to identify which tourists share similar interests. According to these similarities, a recommendation is provided.

A prototype of the mobile recommender system is proposed in (Derakhshan, Parandeh, \& Moradnejad, 2016). The content-based filtering algorithm is used for the provision of recommendations. The prototype is based on the multi-agent architecture where three main agents are used: the user agent (presents information to a user), the recommender agent (provides recommendations) and the activity agent (stores and updates information about various tourist locations). The prototype has been already tested in tourism in Tabriz (Iran), because the mobile application based on personalisation is missing in this city. Persian and English languages are used in the application.

The agent-based model for virtual tourism is proposed in (Pouyan et al., 2006). It can be said that this model is non-traditional, because it connects the multi-agent paradigm, the object oriented Petri nets and tourism. The Petri net (a place/transition net) is a formal graphical language used for modelling a structure and dynamic nature of distributed systems. The system consists of individual agents, i.e., the Secretary agent (receives fundamental information about a tourist (a customer), the Consultation agent receives preferences of a tourist and provide suitable alternatives in terms of destinations, accommodation, how to travel, or costs. These pieces of information are given to the Envoy agent that arranges formalities, e.g., booking of accommodation or an air ticket. The Petri net structure models communication among these mobile agents.

Historical tourism is a special case of tourism where historical locations, persons or events are in the centre of the tourists and tourism. The multi-agent based architecture for the implementation of the recommender system for historical tourism is proposed 
in (Varfolomeyev et al., 2015). Personal recommender service is based on a semantic network (RDF ontology) that represents individual 'objects of the interest' of a tourist and recommends the other 'objects' that can be also interesting for a tourist. The service is focused on the history of Latgale - cultural region in Latvia. The information system Latgales Dati contains the main information about Latgale and is used as a main source for the RDF ontology building. This system contains series of web pages which are transformed to the RDF-triplets representing statements about historical persons and events.

\section{Discussion}

The presented review proves that the multi-agent paradigm is applied in tourism mainly in monitoring, planning, prediction, explanation and recommendation of theories. This conclusion is not so surprising because this spectrum of applications is typical of the multi-agent systems. The multi-agent systems are perceived as the alternative for the exploration of tourism with minimum spending time and costs where useful application can also help with the information overload of the tourists. The multi-agent systems bring the added value into tourism, especially from these points of view:

- complexity modelling: MAS are efficiently applied for the investigation of the complex systems which are often decentralised, dynamic, open, adaptive or able to learn. All these characteristics of complex systems also occur in tourism. In the beginning of the text, it is declared that tourism is a complex system, because it includes a lot of players which are in mutual relations and interactions (tourists and specific destinations, tourists and economic prosperity, weather and attractiveness of a locality for tourists, traffic and tourism in a specific locality, ecological impacts on tourism, possible changes in culture of a locality due to tourism). Tourism is not only complex, but it is a complex adaptive system able to adapt to changing conditions occurring in the environment. The adaptation is an important attribute of survival where the feedback or previous experience influence the behaviour of the system in the future. At present, one can observe how various countries deal with immigrants. Various cultures (multi-agent communities) with own habits, attitudes and behaviour are met in one place where it is often difficult to find consensus for satisfaction of all sides. Countries try to find a balance between sustaining their own identity, habits, social rules, cultural heritage and the willingness to help. Immigration can substantially influence the interest in tourism in individual countries. Speed and efficiency of adaptability in this case depend on political, social and economic conditions and settings. Migration strategies and politics can be also studied with MAS (e.g., Kaur, Kahlon, \& Virk, 2014; or Lin, Carley, \& Cheng, 2016). In addition, conflicts and negotiations can be modelled with the multi-agent systems, often with the use of the game theory. The game theory investigates the interactions among the groups of agents having various aims which are often in contradiction with other agents (Shoham \& Leyton-Brown, 2008). 


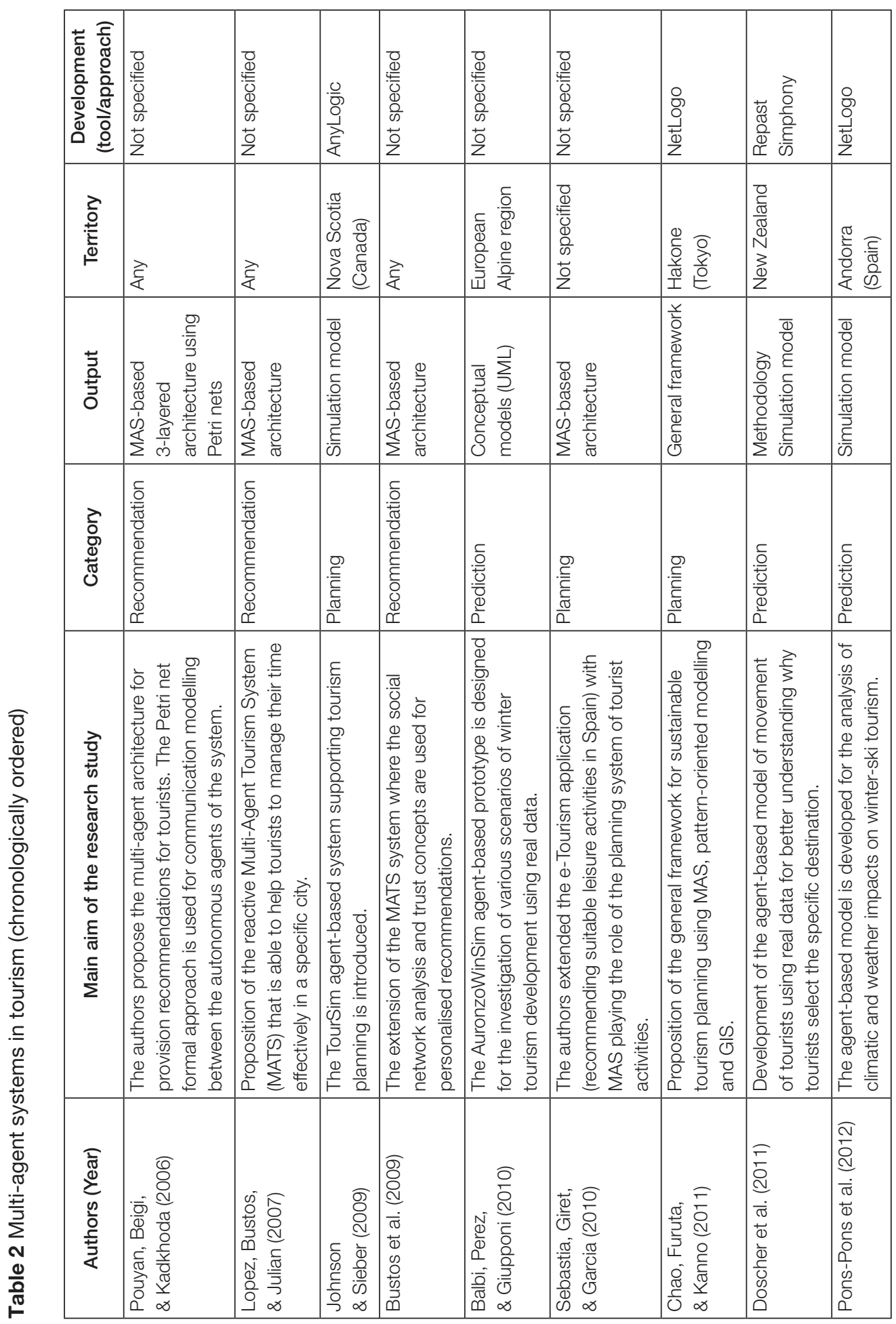




\begin{tabular}{|c|c|c|c|c|c|c|}
\hline $\begin{array}{l}8 \\
8 \\
0 \\
\frac{1}{0} \\
2\end{array}$ & $\begin{array}{l}8 \\
8 \\
\frac{1}{1} \\
\frac{1}{0} \\
\end{array}$ & $\begin{array}{l}z \\
0 \\
\infty \\
\sum \\
\Sigma\end{array}$ & 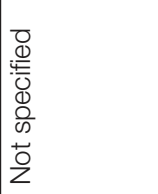 & 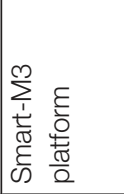 & 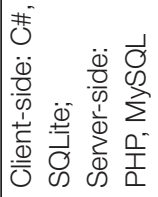 & 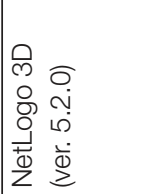 \\
\hline 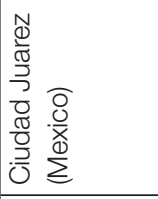 & 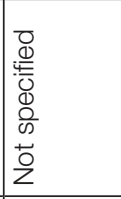 & 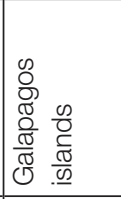 & $\frac{\grave{2}}{\dot{\alpha}}$ & 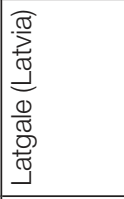 & 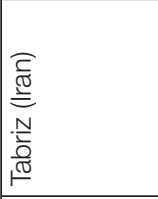 & 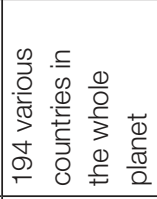 \\
\hline 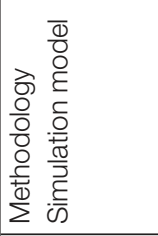 & 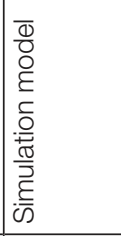 & 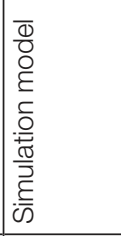 & 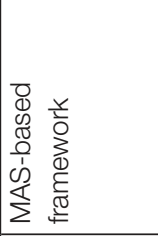 & 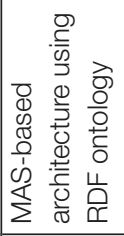 & 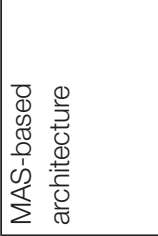 & 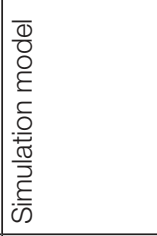 \\
\hline 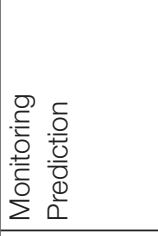 & 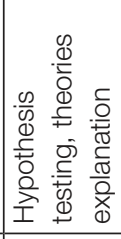 & 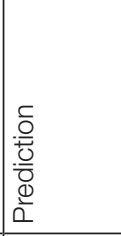 & 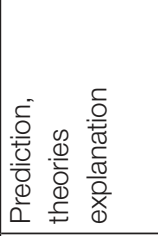 & 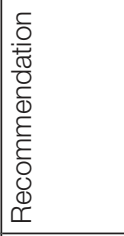 & 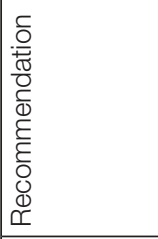 & 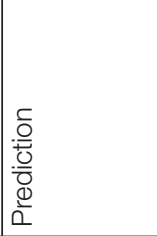 \\
\hline 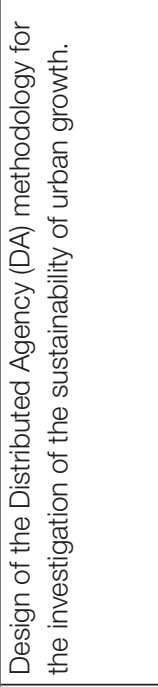 & 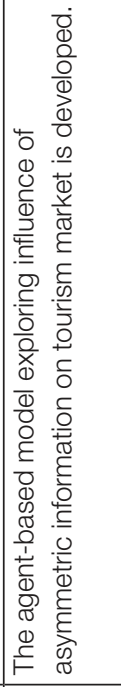 & 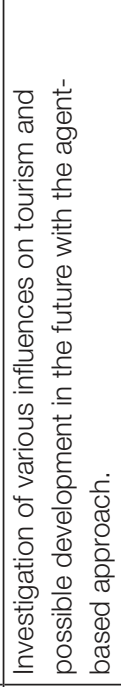 & 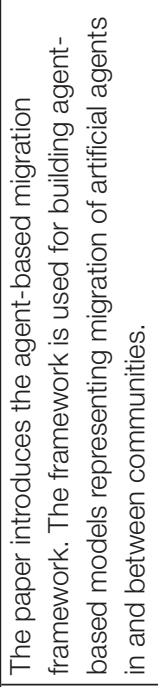 & 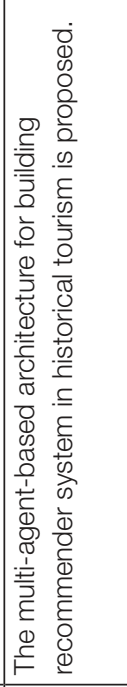 & 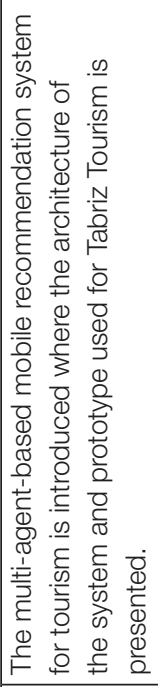 & 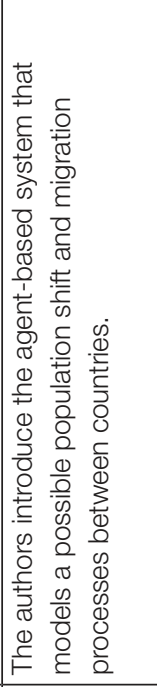 \\
\hline 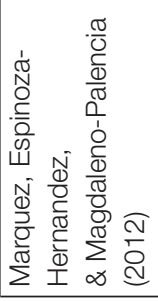 & 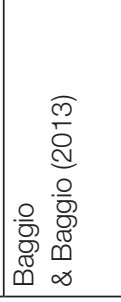 & 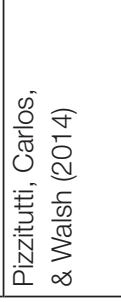 & 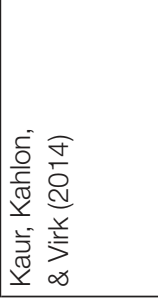 & 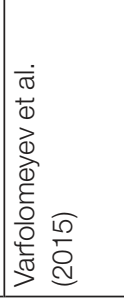 & 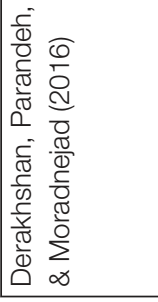 & 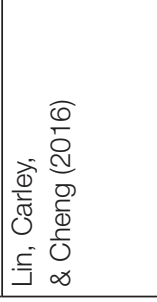 \\
\hline
\end{tabular}


- modelling of the interactions and connections: Various self-interested and pro-social parties are constituents of tourism and it is obvious and natural that the interactions contribute (positively or negatively) to the tourism development. Various forms of coordination and cooperation techniques can be integrated into the MAS-based models, e.g., reactive communication, auction-based interactions, blackboard architecture, contract net-based protocol, negotiations or communication based on speech act theory. More details can be found (e.g., Das \& Gupta, 2014). The complex networks are able to model and visualise the interactions and connections. One can say that tourism can be formally defined as a complex network, see Fig. 2. The relations between tourism and complex networks are introduced (e.g., Scott et al., 2007; Bustos et al., 2009; or Baggio, 2014).

- static and dynamic notion of the system: Tourism is a very complex and dynamic system. The multi-agent systems are mainly focused on the exploration of dynamics of the system and its development. The static aspects of the investigated systems are often represented with various conceptual models which are proposed during the analysis and design of the system. The conceptual models include the most fundamental entities, relations and interactions among them. The conceptual models are then used for programming of the multi-agent system.

- openness and distributiveness of the system: The multi-agent systems are efficient for the modelling of open and distributed systems. These two characteristics are valid also for tourism.

- what-if analysis: The multi-agent computational models can be used for the testing of various scenarios of the system development. The model can consist of different parameters which can be set up with various values. The various settings of the model can be statistically analysed. The hypotheses about possible development of the system can be accepted or rejected.

- emergence: The emergence can be defined as a complex of spontaneous events occurring at macro-levels which cannot be easily deduced from the interactions occurring at micro-levels. Formation of tissues, ripple patterns in a sand dune, snowflakes or building of 'cathedral-like' mounds by termites are typical products of emergence (Charbonneau, Blonder, \& Dornhaus, 2013). Very simple rules can lead to very complex behaviour. Conflicts between supply and demand at global level can produce emergent phenomena where local rules can produce emergent global behaviour.

The agent concept can be easily imagined, e.g., in comparison with differential or difference equations. It depends on the application domain what the agent will represent. In case of tourism, the agent can be a tourist, a destination, accommodation, a car, or a plane. The agent is a natural abstract concept which helps with managing complexity of the system. On the other hand, the exploration of various sides of a complex system with computational models often requires sufficient computational power. If one deal with a very complex system, often thousands or millions of agents are necessary for the computational models. This fact can slow down simulations. This can be a case of tourism where a huge amount of agents is considered, however, it depends on a specific 
purpose of MAS. The distributed multi-agent systems can be a solution (Perrin et al., 2006).

Modelling and simulations often require real data. These data cannot be immediately available or they cannot be available at all. Several reasons can exist: data cannot be acquired from a real system; responsible persons do not want to provide data or data are too expensive. If the computational model contains a huge amount of agents (hundred, thousands), it is often hard to find out something new only on the basis of observing a running model. One has to put right questions, to think about the parameters of the model, to set them up with various values, to receive data from various runs and analysed them to be able to answer initial questions. This activity is time-consuming, however, teamwork can partially deal with that.

The multi-agent systems are a promising approach to testing various scenarios in tourism, its development and sustainability, but the multi-agent systems cannot be used for all situations. The multi-agent-based systems are mainly useful for uncovering often amazing life of complex systems where one cannot study parts of this system in isolation, but in the relation to other parts of the system which are often located in various layers of the systems and where the emergence can occur. As it has been already demonstrated, tourism is perceived as the complex system. MAS should not be applied for the investigation of simple systems containing only a few of components.

\section{Conclusion}

The in-silico modelling is an efficient approach to the understanding of behaviour of the system that is often hardly explored by traditional techniques. The building of models is quite cheap and time-effective because it is not necessary to manipulate with a real system. Modelling is an activity supposing multi-disciplinarity, in which different knowledge is necessary for modelling and simulation. This fact is also true for tourism which can be combined with information and knowledge-based technologies for building sophisticated educational tools, planning systems, recommender systems or applications supporting decision making and performance of what-if analysis for the prediction or explanation of theories. The text presents how tourism can be perceived by complexity science and where one can find the usefulness of the multi-agent system paradigm in tourism and its sustainability. The presented review advocates that the multi-agent systems bring the added value into tourism where especially the modelling, simulation, planning and building recommendation systems can speed up decision making and save time and costs. Thanks to the various MAS-based applications, this bottom-up approach is useful for the investigation of various scenarios of tourism development.

\section{Acknowledgements}

The financial support of the Specific Research Project "Information and knowledge management and cognitive science in tourism" of FIM UHK is gratefully acknowledged. The author would like to thank Jan Hruska for article formatting. 


\section{References}

Axelrod, R. (2003). Advancing the Art of Simulation in the Social Sciences. Journal of the Japanese Society for Management Information Systems. Special Issue on Agent-Based Modeling, 12(3), $16-2$.

Baggio, R. (2005). Complex Systems, Information Technologies, and Tourism: A Network Point of View. Information Technology and Tourism, 8(1), 15-29. DOI: 10.3727/109830506778193850.

Baggio, R. (2014). Complex tourism systems: a visibility graph approach. Kybernetes, 43(3/4), 445461. DOI: $10.1108 / \mathrm{K}-12-2013-0266$.

Baggio, R., \& Baggio, J. A. (2013). Modeling Information Asymmetries in Tourism. In M. Kozak et al. (Eds.), Tourism Marketing: On Both Sides of the Country (pp. 154-174). Cambridge: Scholars Publishing.

Balbi, S., Perez, P., \& Giupponi C. (2010). A spatial agent-based model to explore scenarios of adaptation to climate change in an alpine tourism destination. In A. Ernst \& S. Kuhn (Eds.), 3rd World Congress on Social Simulation (pp. 700-707). Retrieved from https:// papers.ssrn.com/ sol3/papers.cfm?abstract_id=1566861.

Borshchev, A. (2013). The Big Book of Simulation Modeling: Multimethod Modeling with AnyLogic 6. AnyLogic North America. ISBN 978-0989573177.

Bustos, F., López, J., Julián, V., \& Rebollo, M. (2009). STRS: Social Network Based Recommender System for Tourism Enhanced with Trust. In Proceedings of the International Symposium on Distributed Computing and Artificial Intelligence (pp. 71-79). Berlin Heidelberg: Springer-Verlag. DOI: 10.1007/978-3-540-85863-8_10.

Chao, D., Furuta, K., \& Kanno, T. (2011). A Framework for Agent-Based Simulation in Tourism Planning. Proceedings of the Human-Computer Interaction. Towards Mobile and Intelligent Interaction Environments (Part III, pp. 280-287). Berlin Heidelberg: Springer-Verlag. DOI: 10.1007/978-3-642-21616-9_31.

Charbonneau, D., Blonder, B., \& Dornhaus, A. (2013). Social Insects: A Model System for Network Dynamics. In P. Holme \& J. Saramäki (Eds.), Temporal Networks - Understanding Complex Systems (pp. 217-244). Berlin Heidelberg: Springer-Verlag. DOI: 10.1007/978-3-642-36461-7_11.

Collier, N., Howe, T., \& North, M. (2003). Onward and upward: The transition to Repast 2.0. In K. Carley (Ed.), Proceedings of the first annual North Americal Association for Computational Social and Organisational Science conference.

Das, D., \& Gupta, S. (2014). Communication, Cooperation, Coordination and Cognition of a Multi-Agent System - A Literature Survey. International Journal of Latest Research in Science and Technology, 3(2), 147-155. ISSN (Online): 2278-5299.

Derakhshan, F., Parandeh, M., \& Moradnejad, A. (2016). An Agent-Based Mobile Recommender System for Tourisms. In Proceedings of 8th Research World International Conference, Berlin (pp. 30-34). ISBN 978-93-85973-05-5.

Doscher, C., Moore, K., Smallman, C., Wilson, J., \& Simmons, D. G. (2011). An agent-based model of tourist movements in New Zealand: implications for spatial yield. In 19th International Congress on Modelling and Simulation. Modelling and Simulation (pp. 2908-2913). Society of Australia and New Zealand. ISBN 978-0-9872143-1-7.

Durlauf, S. N. (1998). What Should Policymakers Know About Economic Complexity? The Washington Quarterly, 21, 155-165. Retrieved from https://www.santafe.edu/media/workingpapers/97-10-080.pdf.

Foote, R. (2007). Mathematics and complex systems. Science, 318, 410-412. DOI: 10.1126/science.1141754 
Holloway, J. C., Humphreys, C., \& Davidson, R. (2009). The Business of Tourism. Pearson Education Limited, 8th ed. ISBN 978-0-273-71710-2.

Horling, B., \& Lesser, V. (2004). A Survey of Multi-Agent Organizational Paradigms. The Knowledge Engineering Review, 19(4), 281-316. DOI: 10.1017/S0269888905000317.

Johnson, P. A., \& Sieber, R. E. (2009). Agent-based modelling: A Dynamic Scenario Planning Approach to Tourism PSS. In S. Geertman \& J. Stillwell (Eds.), Planning support systems: Best practices and new methods (pp. 211-226). Berlin Heidelberg: Springer-Verlag. DOI: 10.1007/978-14020-8952-7_11.

Kaur, H., Kahlon, K. S., \& Virk R. S. (2014). Migration Dynamics in Artifical Agent Societies. International Journal of Advanced Research in Artificial Intelligence, 3(2), 39-47. DOI: 10.14569/ IJARAI.2014.030208.

Kravari, K., \& Bassiliades, N. (2015). A Survey of Agent Platforms. Journal of Artificial Societies and Social Simulation, 18(1). Retrieved from http://jasss.soc.surrey.ac.uk/18/1/11.html. DOI: 10.18564/jasss.2661.

Lin, L., Carley, K. M., \& Cheng, S. F. (2016). An Agent-Based Approach to Human Migration Movement. In T. M. K. Roeder, P. I. Frazier, R. Szechtman, E. Zhou, T. Huschka, \& S. E. Chick (Eds.), Proceedings of the 2016 Winter Simulation Conference (pp. 3510-3520). Arlington: Research Collection School of Information Systems. DOI: 10.1109/WSC.2016.7822380.

Lopez, J. S., Bustos, F. A., \& Julian V. (2007). Tourism services using agent technology: A multiagent approach. In INFOCOMP Journal of Computer Science (Special edition, pp. 51-57).

Luke, S., Cioffi-Revilla, C., Panait, L., Sullivan, K., \& Balan, G. (2005). Mason: A multiagent simulation environment. Simulation: Transactions of the Society for Modeling and Simulation, Simulation, 82(7), 517-527. DOI: 10.1177/0037549705058073.

Marquez, B. Y., Espinoza-Hernandez, I., \& Magdaleno-Palencia, J. S. (2012). Sustainable System Modelling for Urban Development Using Distributed Agencies. In C. Ghenai (Ed.), Sustainable Development - Policy and Urban Development - Tourism, Life Science, Management and Environment. InTech. DOI: $10.5772 / 28898$.

Mills, A. (2010). Complexity Science: An introduction (and invitation) for actuaries. Society of Actuaries. Retrieved from https://www.soa.org/files/research/projects/research-complexity-report.pdf.

Perrin, D., Ruskin, H. J., Burns, J., \& Crane, M. (2006, May). An agent-based approach to immune modelling. In International Conference on Computational Science and Its Applications (pp. 612-621). Berlin Heidelberg: Springer-Verlag. DOI: 10.1007/11751540_65.

Pizzitutti, F., Carlos, F. M., \& Walsh, S. J. (2014). Modelling Tourism in the Galapagos Islands: An Agent-Based Model Approach. Journal of Artificial Societies and Social Simulation, 17(1), Retrieved from http://jasss.soc.surrey.ac.uk/17/1/14.html. DOI: 10.18564/jasss.2389.

Pons-Pons, M., Johnson, P. A., Rosas-Casals, M., Sureda, B., \& Jover, È. (2012). Modeling climate change effects on winter ski tourism in Andorra. Climate research, 54(3), 197-207. DOI: 10.3354/cr01117.

Pouyan, A. A., Beigi, A. H., \& Kadkhoda, M. (2006). An Agent-Based Model for Virtual Tourism Using Object Petri Nets. In Proceedings of the 5th WSEAS Int. Conf. on Circuits, Systems, Electronics, Control and Signal Processing (pp. 149-154). Dallas: USA. ISBN: 960-8457-55-6.

San Miguel, M., Johnson, J. H., Kertesz, J., Kaski, K., Díaz-Guilera, A., MacKay, R. S., \& Helbing, D. (2012). Challenges in complex systems science. arXiv:1204.4928v1 [nlin.AO]. The European Physical Journal Special Topics, 214(1), 245-271. Retrieved from https://arxiv.org/abs/1204.4928.

Scott, N. R., Cooper, C. P., \& Baggio, R. (2007). Use of network analysis in tourism research. In L. Andreu, J. Gnoth, \& M. Kozak (Eds.), Advances in Tourism Marketing Conference, Valencia: Spain. Retrieved from http://www.iby.it/turismo/papers/baggio-ATMC2007-1.pdf. 
Sebastia, L., Garcia, I., Onaindia, E., \& Guzman, C. (2009). e-Tourism: a tourist recommendation and planning application. International Journal on Artificial Intelligence Tools, 18(5), 717-738. DOI: 10.1109/ICTAI.2008.18.

Sebastia, L., Giret, A., \& Garcia I. (2010). A Multi Agent Architecture for Tourism Recommendation. In Proceedings of the 8th International Conference on Practical Applications of Agents and Multiagent Systems (pp. 547-554). Berlin Heidelberg: Springer-Verlag. DOI: 10.1142/S0218213009000378.

Sheard, S., Cook, S., Honour, E., Hybertson, D., Krupa, J., McEver, J., \& Singer, J. (2015). A Complexity Primer for Systems Engineers. INCOSE Complex Systems Working Group White Paper. Retrieved from: https://www.aiaa.org/uploadedFiles/Events/Complexity\%20Primer\%20 for\%20SE\%20July\%202015.pdf.

Shoham, Y., \& Leyton-Brown, K. (2008). Multiagent Systems: Algorithmic, Game-Theoretic, and Logical Foudnations. Cambridge: University Press, 1st ed. ISBN 978-0521899437

Smeral, E. (1993). Aspects to justify pubic tourism promotion: An economic perspective. Tourism Review, 61(3), 6-14. DOI: 10.1108/eb058474.

United Nations Environment Programme, \& United Nations World Tourism Organisation. (2005). Making Tourism More Sustainable - A Guide for Policy Makers. UNEP, UNWTO. http://www. unep.fr/scp/publications/details.asp?id=DTI/0592/PA. Accessed August 2005

Varfolomeyev, A., Korzun, D., Ivanovs, A., Soms, H., \& Petrina, O. (2015). Smart space based recommendation service for historical tourism. Procedia Computer Science, 77, 85-91. DOI: 10.1016/j.procs.2015.12.363.

Vig, L., \& Adams, J. A. (2006). Multi-Robot Coalition Formation. IEEE Transactions On Robotics, 22(4), 637-649. DOI: 10.1109/TRO.2006.878948.

Weiss, G. (2000). Multi-agent Systems: A Modern Approach to Distributed Artificial Intelligence. England, Cambridge Massechusetts: MIT Press. ISBN 978-0262731317.

Wilensky, U. (1999). NetLogo (and NetLogo User Manual). Center for Connected Learning and Computer-Based Modeling, Northwestern University. Retrieved from http://ccl.northwestern.edu/ netlogo/.

Woodridge, M., \& Jennings, N. R. (1995). Intelligent agents: theory and practice. The Knowledge Engineering Review, 10(2), 115-152.

Wooldridge, M. J. (2013). Intelligent Agents. In G. Weiss (Ed.), Multi-Agent Systems (2nd edn., pp. 3-50). England: MIT Press. ISBN 978-0-262-01889-0. 